\title{
Shifting the half wave dipole antenna resonance using EBG structure
}

\author{
El Ghabzouri Mohammad, Abdenacer Es-Salhi \\ LETAS Dept. of physics, Faculty of Sciences, Mohamed I \\ University \\ Oujda, Morocco \\ elghabzouri.mad@gmail.com
}

\author{
Paulo M. Mendes \\ Dept. of Industrial Electronics, University of Minho \\ Guimarães, Portugal
}

\begin{abstract}
In this paper, the main motivation is to introduce a new behavior of the electromagnetic band gap (EBG) structures, it is a significant shifting the resonance frequency down of the dipole antennas, this very interesting and useful technique led to low profile, in addition to performance enhancement of dipole antennas, either on return loss or radiation pattern. Also among this EBG structure an investigation on specific absorption rate (SAR) is shown. The used dipole antenna is resonating around 3.5 GHz (part of $4 \mathrm{G}$ bands), then by using this technique we could shift the working frequency of the same dipole antenna to $2.8 \mathrm{GHz}$ worldwide interoperability for microwave access (WiMAX), this new resonance is $\mathbf{8 0 \%}$ lower compared with the normal resonance without any structure. The principle of this new technique still valid with other frequency, depending on the frequencies that we would like to shift the working frequency between.
\end{abstract}

Keywords-Half wave dipole antenna; radiation pattern; electromagnetic band gap (EBG); specific absorption rate (SAR).

\section{INTRODUCTION}

Many significant works showing the importance of electromagnetic band gap (EBG) structures, firstly in optic area and then in wireless domain. The purpose of the present work is to show how it is efficient to have low profile dipole antenna using those kinds of structures. The Sievenpiper structure which is mushroom like was the beginning of this revolution in optics [1], thereafter one of the famous Sami and Yang works describes how successful it is to reduce a coupling between two patch antennas above this EBG mushroom like structure [2], and some results, recently published present the same behavior of these structures, which manifest in mutual coupling reduction of waveguide slot array antennas (WSAA) [3]. Many of succeeded works try to bring some modifications to this basic Sievenpiper structure, to achieve an enhancement to different antenna parameters [4-5]. Others show specific absorption rate (SAR) investigation by using square and circular patch cells [6-7]. In fact, some recent works have shown that the choice of EBG structures integrated with antennas could be a good alternative to enhance the directivity of monopole, dipole and patch antennas [8].

The present paper illustrate a particular and efficient technique to shift down the half wave dipole antenna resonance frequency, This shifting to low frequency is made by using a standard mushroom like EBG, and the shifted resonance frequency was driven by using our fabricated EBGCS structure presented in [9]. In fact, the EBG structures can be also used to drive the central frequency of the antenna, by using some specific equations [10]. Also the radiation pattern improvement due to surface wave suppression is showed among this work.

\section{CHARACTERISATION BAND GAP METHODS}

There are many methods to study this kind of electromagnetic structure, and identifying their band gaps.

We can mention here a dispersion diagram method, which analyze the propagation of electromagnetic waves above the structure by calculating the Eigen values inside the Brillouin zone, the reflection phase method and direct transmission method (DTM). In the following paragraphs, some details describing these methods are detailed. Then we apply the suspended method line to our proposed configuration; cause its practical realization to be compared with feature measurements.

\section{A. Reflection phase methode}

In this paragraph, we start with one of the most useable methods. With the reflection phase method we can easily characterize our unit cell and then identify the band gap around the resonance frequency. To extract this reflection phase curve, the unit cell was modeled and excited by a wave guide port at $Z_{\max }$ position which is ten time great than the substrate thickness $h$. We make sure that the boundary conditions $X_{\min }$ and $X_{\max }$ defined as electric walls, $Y_{\min }$ and $Y_{\max }$ defined as magnetic walls, and $Z_{\text {min }}$ defined as electric walls.

\section{B. Direct transmission line method}

This method based on entire modeling of one dimension of the unit cell. The proposed EBG structure is constituted by $6 * 4$ cells, which mean four cells by line. Hence we transmit a planar wave through the line of cells by one side, and we calculate the transmission parameter $S_{21}$ in the other side of the line, in order to determine the prohibited band above our structure. In this method, we have to follow some boundary conditions, because this method considers a line of cells as a TEM wave guide, which mean that the right and the left side are chosen as magnetic walls. In the other hand the bottom and 
top side are chosen as electric walls. Finally the two rest sides are kept as open boundaries.

\section{Dispersion diagram method}

This identification technique is more used by programming FDTD (Finite Deference Time Domain) method. But also we can calculate the dispersion diagram by a special solver. Eigen mode solver is another way to determine a band diagram, and then we identify our band gap, after that we optimizing our cell to operate at the work frequency of our antenna. The figure below illustrates the design of the unit cell.

The method consist to define a periodic boundary conditions along $\mathrm{U}$ axis $\left(\mathrm{X}_{\min }\right.$ and $\left.\mathrm{X}_{\max }\right)$, and same thing to $\mathrm{V}$ axis $\left(\mathrm{Y}_{\min }\right.$ and $\left.\mathrm{Y}_{\max }\right)$, and finally we define $\mathrm{W}$ axis $\left(\mathrm{Z}_{\min }, \mathrm{Z}_{\max }\right)$ as an $E_{t}=0$ (tangential electric field) for TE mode calculation or $\mathrm{H}_{\mathrm{t}}=0$ (tangential magnetic field) for TM mode calculation.

The main difficulty of the previous three methods is not practical to be realized for measurement and comparison, in opposite to the following method, which is simple and efficient to compute the right S21 of the structure.

\section{Suspended line method}

The concept of this method is based on designing the whole EBG structure, and then we insert under the structure a transmission line between the two extreme parts of the studied structure, finally we feed the transmission line by two wave guide port to transmit and receive the waves [11].

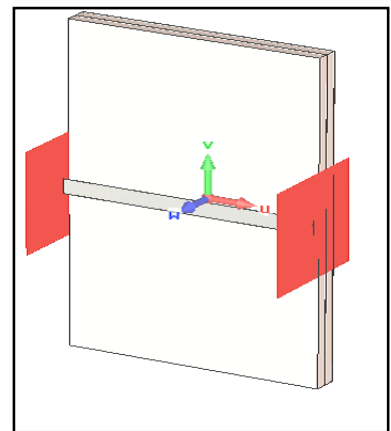

Fig. 1. Design of the suspended line method.

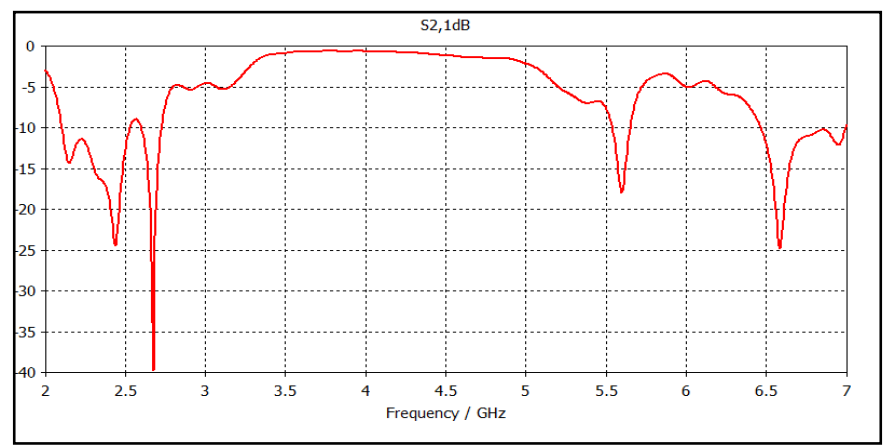

Fig. 2. The transmission coefficient S21 showing the band gap of the used structure
Beside some narrow band gaps, at least less than $7 \mathrm{GHz}$. the computed transmission coefficient S21 illustrated in Fig.2 shows almost no stop band at $-20 \mathrm{~dB}$ between 2.7 to $3.7 \mathrm{GHz}$, the dimensions of this EBG structure were chosen to have a transparent structure around these frequencies, which is our zone of interest in this work. Therefore the present structure is modeled to shift the resonance frequency from 3.5 to $2.8 \mathrm{GHz}$, in favor of some particular application, like a balance between fourth generation band $(4 \mathrm{G})$ around $3.5 \mathrm{GHz}$ and WiMAX application at $2.8 \mathrm{GHz}$ for hand cell phones.

\section{Dipole ANTENNA WITH AND WITHOUT EBG STRUCTURE}

\section{A. Return losses results and discussion}

In the following section we present some details concerning the half wave dipole antenna design, this dipole antenna modeled to be radiating around fourth generation 3.5 $\mathrm{GHz}$ band. The length "l" of dipole antenna is approximately a half wave length $l=\lambda / 2, \lambda$ present the wave length on free space at the center frequency, the input impedance of the half wave dipole antenna was set at $73 \mathrm{ohm}$. The antenna radiate at $3.5 \mathrm{GHz}$ in free space. Then we will use the antenna in front of the EBG structure background Fig.3, the results in Fig 5 show a very important shifting on the resonance frequency, this new configuration (antenna in front of the EBG mushroom like) shift the resonance frequency down to $2.8 \mathrm{GHz}$, which the IEEE standard for WiMAX application, the band size of shifting down is $0.7 \mathrm{GHz}$ which is $80 \%$ lower from the central frequency of the dipole antenna without EBG structure.

Both the substrate and superstrat material was chosen as Rogers RO4350 with dielectric constant 10.2 with the standard fabricated thickness $1.27 \mathrm{~mm}$. The figures 4 and 5 shows the return losses of both the dipole antenna in free space and mounted with EBG structure.

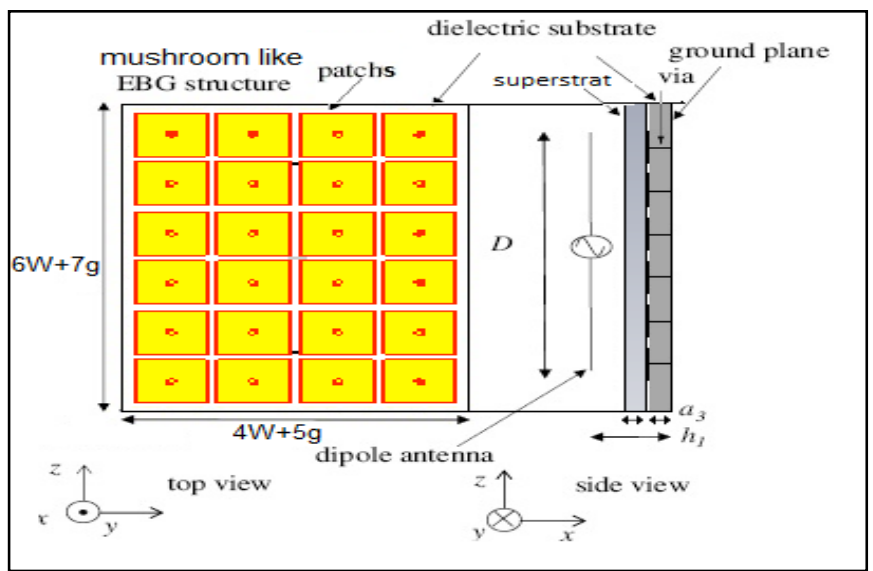

Fig. 3. The design of dipole antenna and EBG structure mounted with superstrat.

$\mathrm{W}=6.2 \mathrm{~mm} ; \mathrm{g}=0.5 \mathrm{~mm} ; \mathrm{a} 3=1.27 \mathrm{~mm} ; \mathrm{h}=2 * \mathrm{a} 3+0.5 \mathrm{~mm} ; \mathrm{D}=40 \mathrm{~mm}$ 


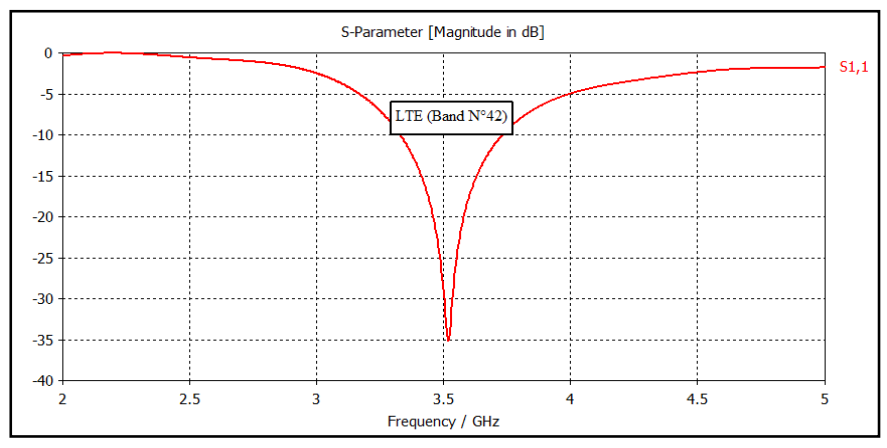

Fig. 4. return loss of the dipole antenna without EBG structure.

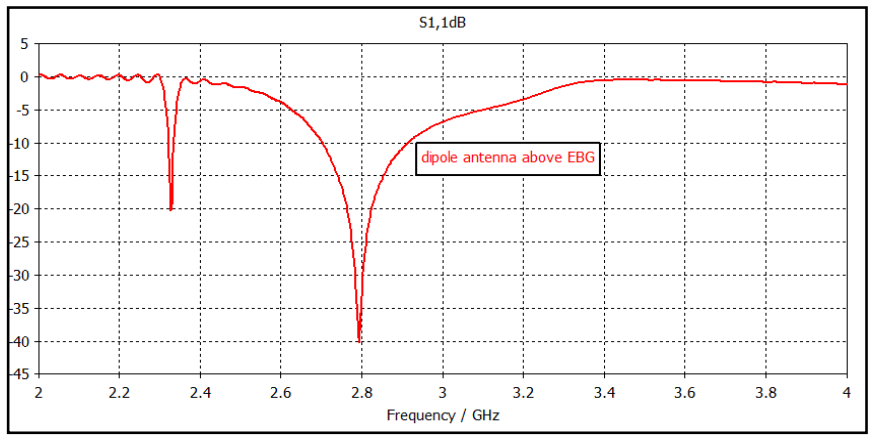

Fig. 5. Return loss of the same dipole antenna with the new working frequency $2.8 \mathrm{GHz}$ when used with the proposed $\mathrm{EBG}$.

\section{B. Rediation pattern results and discussion}

The fowling section illustrates the comparison results of the $3 \mathrm{D}$ radiation pattern, together with the horizontal and vertical cut. After shifted the antenna working frequency down from $3.5 \mathrm{GHz}$ to $2.8 \mathrm{GHz}$, when using the EBG structure. Now the results of this new configuration should be compared with a half wave dipole antenna designed to be radiating around $2.8 \mathrm{GHz}$. The half wave dipole antenna has an Omnidirectional diagram with $1.96 \mathrm{dBi}$ of its directivity Fig.2. Therefore the $3 \mathrm{D}$ radiation pattern of the proposed configuration of the half wave dipole antenna with EBG structure is showed in Fig.3.

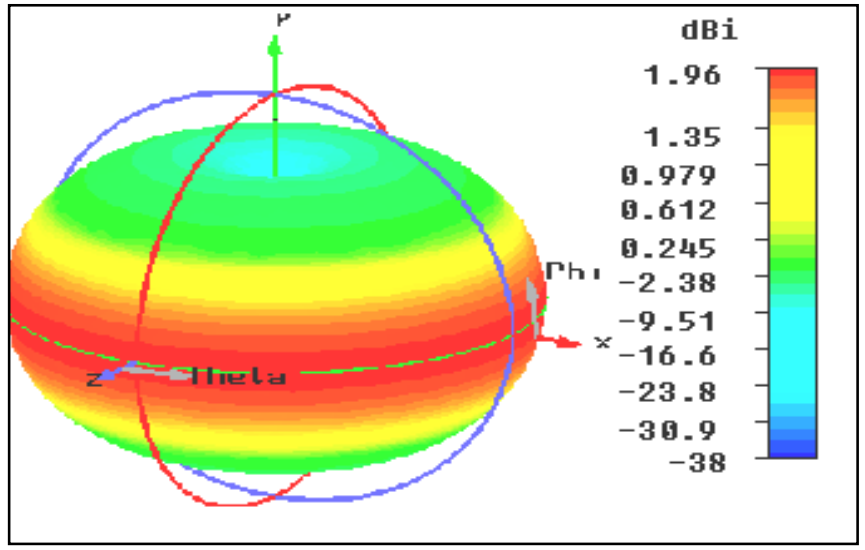

Fig. 6. The far field radiation pattern of the half wave dipole antenna at $2.8 \mathrm{GHz}$

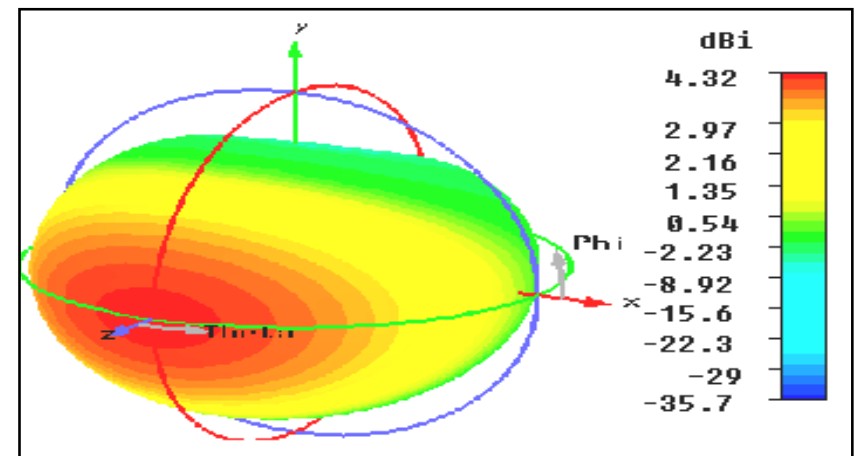

Fig. 7. The far field radiation pattern of the half wave dipole antenna mounted with mushroom like EBG shifted from 3.5 to $2.8 \mathrm{GHz}$.

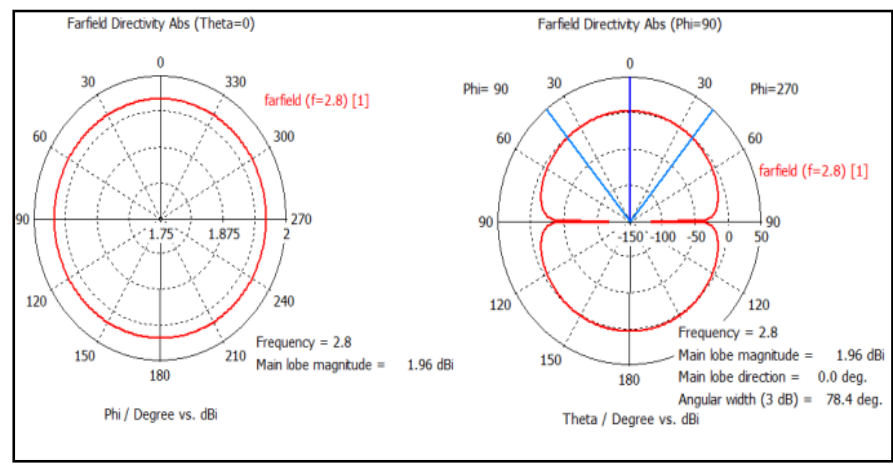

Fig. 8. Vertical and horizontal cut of radiation pattern of dipole antenna.

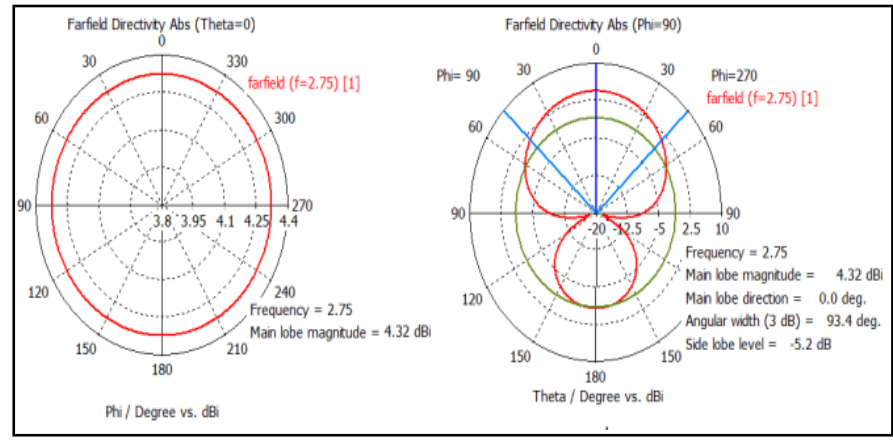

Fig. 9. Vertical and horizontal cut of radiation pattern of dipole antenna with EBG structure.

This proposed configuration, in addition, to low profile achievement, which manifest in shifting the resonance frequency from $3.5 \mathrm{GHz}$ ( $4 \mathrm{G}$ band) to $2.8 \mathrm{GHz}$ (WiMax), led to a very good radiation pattern compared with the reference dipole antenna, as the figures show. the maximum directivity was improved by more than $100 \%$ (from $1.96 \mathrm{dBi}$ in Fig. 6 to $4.32 \mathrm{dBi}$ in Fig.7), but the improvement was only on the front half space, the directivity on back half space was a bit poor also compared with the reference dipole antenna. Generally the results agreeable than what we were expecting.

\section{SAR INVESTIGATION}

The specific absorption rate is a very important parameter to study for in any antenna before its realization, because exposing our bodies in antennas, means that the antennas should satisfy international requirements of SAR. Basically 
SAR is presenting the quantity of absorbed power in our biological tissues. The Fig.8, show the far field radiation pattern of the half wave dipole antenna, mounted with the proposed mushroom like EBG, shifted from 3.5 to $2.8 \mathrm{GHz}$, when used with the human head phantom. So we can see that the human head increased the maximum directivity from 4.32 $\mathrm{dBi}$ to $6.91 \mathrm{dBi}$, which is understandable if we take in account the reflected power when used the antenna nearby the human head.

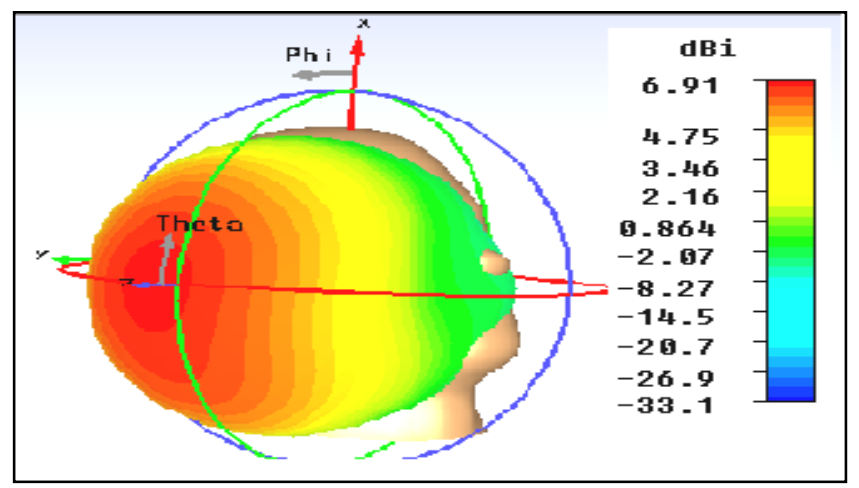

Fig. 10. The far field radiation pattern at $2.8 \mathrm{GHz}$.

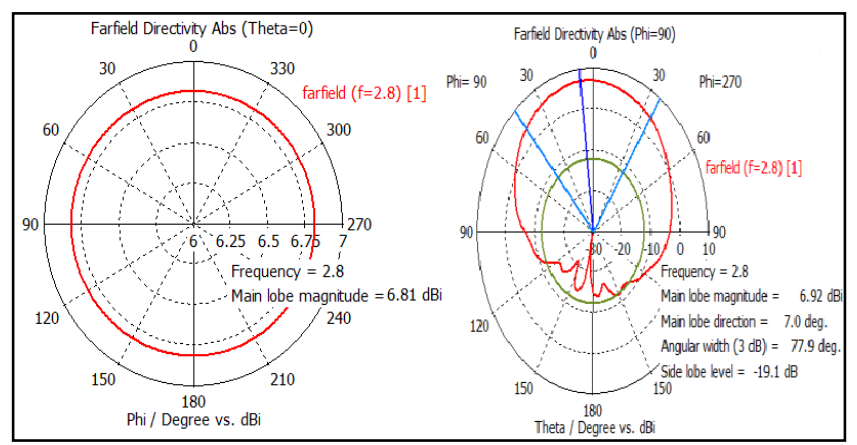

Fig. 11. Vertical and horizontal cut of the influenced radiation pattern of dipole antenna with EBG structure close to the human head.

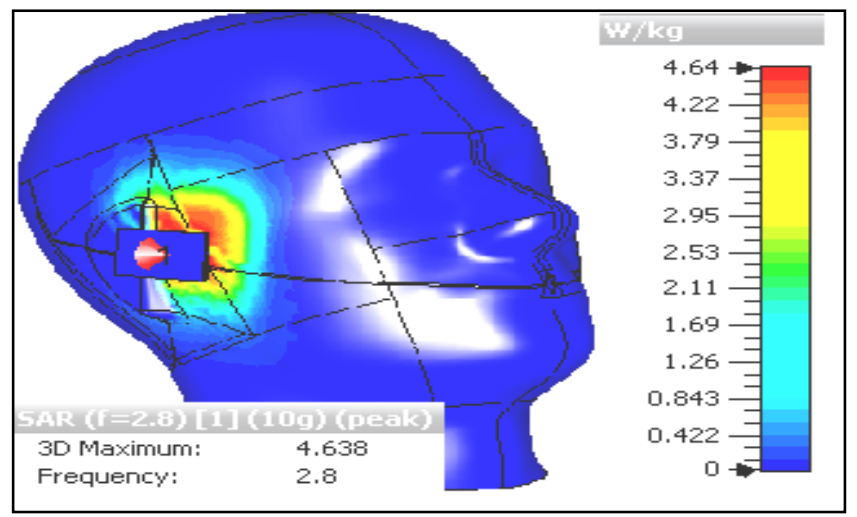

Fig. 12. SAR cartography at $2.8 \mathrm{GHz}$ averaged on $10 \mathrm{~g}$.

Finally we have computed the absorbed power (SAR) inside the tissues, the liquid characteristics was chosen to simulate the human tissues at $2.8 \mathrm{GHz}$, the power was normalized to 1 watt, and the distance between the phantom and the structure was set at $10 \mathrm{~mm}$.

\section{CONCLUSION}

This work resumes another efficient and important use of EBG structure in antenna community, this new technique consist to shift to low frequency the resonating frequency of antennas, this shifting was achieved by using a special design of EBG structure, the half wave dipole antenna was shifted from $3.5 \mathrm{GHz}$ to $2.8 \mathrm{GHz}$, which present $80 \%$ of miniaturization of antenna, which led to the contribution to low profile antenna for cell phones, at the same time we could balance between $4 \mathrm{G}$ resonance and WiMAX resonance with only on antenna. Also we have been presented that this technique useable to improve a high efficiency antenna, using the EBG structure. Which manifest in very low return loss of dipole antenna, its $220 \mathrm{MHz}$ bandwidth, in addition to the improvement of the effectiveness of the radiation pattern, by the proposed configuration of EBG structure, finally we have computed the absorbed power (SAR) inside the human head phantom.

\section{References}

[1] D. Sievenpiper, L. Zhang, R. F. J. Broas, N. G. Alexópolous, and E. Yablanovitch, "High-Impedance Electromagnetic Surfaces with a Forbidden Frequency Band," IEEE Transactions on Microwave Theory and Techniques, Vol.47, No.11, November 1999, pp. 2059-2074.

[2] Fan Yang, Member, IEEE, and Yahya Rahmat-Samii, Fellow, IEEE "Microstrip Antennas Integrated With Electromagnetic Band-Gap (EBG) Structures: A Low Mutual Coupling Design for Array Applications," IEEE Transactions On Antennas and Propagation, vol. 51,no.10, October 2003.

[3] Siamak Ebadi, and Abbas Semnani, "Mutual coupling reduction in waveguide slot array antennas using electromagnetic band gap (EBG) structures." IEEE Antennas and Propagation Magazine, vol. 56,no.3, June 2014.

[4] N. Elsheakh, H. A. Elsadek, and E. A. Abdallah, "investigated new embedded shapes of electromagnetic bandgap structures and via effect for improved microstrip patch antenna performanced," In Progress In Electromagnetics Research B, Vol. 20, 91_107, 2010.

[5] Seungbae Park, Cheolbok Kim, Youngho Jung, Hosang Lee, Dongki Cho,Munsoo Lee, "Gain enhancement of amicrostrip patch antenna using a circularly periodic EBG structure and air layer," In Int.J.Electron.Commun.(AEÜ) 64(2010)607-613

[6] Kwok-Hung Chan, Member, IEEE, Ryo Ikeuchi, and Akimasa Hirata, Senior Member, IEEE "Effects of phase difference in Dipole phasedarray antenna above EBG substrates on SAR," IEEE Antennas Wireless Propag. Lett., vol. 12, 2013.

[7] Ryo Ikeuchi and Akimasa Hirata, Senior Member, "Dipole antenna above EBG substrate for local SAR reduction," IEEE Antennas Wireless Propag. Lett., vol. 10, 2011.

[8] Silvio Ceccuzzi, Lara Pajewski, Cristina Ponti, and Giuseppe Schettini, "Directive EBG Antennas: A Comparison Between Two Different Radiating Mechanisms," IEEE Transactions On Antennas And Propagaton, Vol. 62, NO. 10, october 2014.

[9] Mohammad El Ghabzouri, A. Es Salhi, P. M. Mendes, "Dual band antenna size reduction using EBG structure," Mediterranean Conference on Information \& Communication Technologies'2015 May 7-9, 2015 Saïdia, Morocco, unpublished.

[10] Mohammad El Ghabzouri, A. Es Salhi, P. M. Mendes, "Dual band antenna size reduction using EBG structure,"IEEE Mediterranean microwave symposuim 2015 (MMS2015) 'leece, italy, unpublished.

[11] M. S. Alam, M. T. Islam, N. Misran, "A novel compact split ring slotted electromagnetic band gap structure for microstrip patch antenna performance enhancement," Progress In Electromagnetics Research, Vol. 130, 389-409, 2012. 